\title{
BMJ Open Protection motivation theory screening tool for predicting chronic low back pain rehabilitation adherence: analysis of a randomised controlled trial
}

\author{
Patrick J Owen (D) , ${ }^{1}$ Luana C Main (D) , ${ }^{1}$ Clint T Miller (D) , ${ }^{1}$ Jon J Ford (D) ,2,3 \\ Andrew J Hahne (D) ,2 Daniel L Belavy (D) ${ }^{1,4}$
}

To cite: Owen PJ, Main LC, Miller CT, et al. Protection motivation theory screening tool for predicting chronic low back pain rehabilitation adherence: analysis of a randomised controlled trial. BMJ Open 2022;12:e052644. doi:10.1136/ bmjopen-2021-052644

- Prepublication history for this paper is available online. To view these files, please visit the journal online (http://dx.doi org/10.1136/bmjopen-2021052644).

Received 22 April 2021 Accepted 18 January 2022

\section{Check for updates}

(c) Author(s) (or their employer(s)) 2022. Re-use permitted under CC BY-NC. No commercial re-use. See rights and permissions. Published by BMJ.

${ }^{1}$ Institute for Physical Activity and Nutrition (IPAN), Deakin University, Geelong, Victoria, Australia

'Low Back Research Team, College of Science, Health \& Engineering, La Trobe University, Bundoora, Victoria, Australia

${ }^{3}$ Advanced HealthCare, Boronia,

Victoria, Australia

${ }^{4}$ Department of Applied

Health Sciences, Division of

Physiotherapy, Hochschule für Gesundheit (University of Applied Sciences), Bochum, Germany

Correspondence to

Dr Patrick J Owen;

p.owen@deakin.edu.au

\section{ABSTRACT}

Objective To explore a protection motivation theory screening tool for predicting rehabilitation adherence. Design Analysis of a randomised controlled trial. Setting An exercise physiology and physiotherapist clinic. Participants Patients with chronic low back pain $(n=40)$. Interventions General strength and conditioning (GSC) compared with motor control and manual therapy. Primary and secondary outcome measures: predicting patient adherence to supervised sessions and dropout using the Sports Injury Rehabilitation Beliefs Scale, sevenitem barriers checklist and Positive and Negative Affect Schedule and Sports Injury Rehabilitation Adherence Scale. Results Average attendance was $77 \%$ (motor control and manual therapy) and $60 \%$ (GSC) with eight dropouts. No Sports Injury Rehabilitation Adherence Scale values other than 5 across all three components were recorded. Treatment efficacy $(p=0.019)$, self-efficacy $(p=0.001)$, rehabilitation value $(p=0.028)$ and injury severity $(p=0.002)$ positively correlated with susceptibility (the extent of vulnerability to having health problems from not taking action). Rehabilitation value positively correlated with self-efficacy $(p=0.005)$. Injury severity positively correlated with rehabilitation value $(p=0.011)$. The final model for number of cancellations included rehabilitation value only and accounted for approximately $12 \%$ of variance $(p=0.033)$.

Conclusions Perceived value of rehabilitation should be considered by clinicians in the rehabilitation setting to improve treatment adherence in patients with chronic low back pain.

Trial registration number ACTRN12615001270505.

\section{INTRODUCTION}

Worldwide, low back pain (LBP) is the most common non-communicable disease and leading cause of disability. ${ }^{12}$ While chronic low back pain (CLBP), defined as pain lasting 12 weeks or longer, ${ }^{3}$ represents approximately $20 \%$ of the spectrum of $\mathrm{LBP},{ }^{4}$ it is responsible for $80 \%$ of cost of managing this condition. ${ }^{5}$ Most recent estimates in Australia showed direct and indirect costs of LBP were in excess of $\$ 9$ billion per year. ${ }^{6}$ As the structural source
Strengths and limitations of this study

- This study was strengthened by the pragmatic design of the interventions, whereby both treatments reflected interventions used in the management of chronic low back pain.

- Adherence with home-based components of these interventions was not considered, which may have influenced face-to-face session adherence.

- As the population examined had chronic pain, dropout status and/or adherence behaviours may have been influenced by fluctuations in pain that were not detected during routine assessment of pain intensity.

- Sports Injury Rehabilitation Adherence Scale data were collected only in the general strength and conditioning group; hence, conclusions drawn on its potential limitations do not also expand to motor control and manual therapy group.

- Prediction models were considered retrospectively; therefore, we were unable to conclude whether addressing significant independent variables prospectively influences adherence behaviours.

of CLBP cannot be definitively identified in $90 \%$ of cases, the term non-specific has been recommended. ${ }^{7}$

Exercise training interventions designed to reduce the burden of disability in those with non-specific CLBP have been investigated with varying effectiveness. ${ }^{89}$ The variations in outcome may in part be explained by adherence (extent to which one corresponds with the agreed recommendations from a treating clinician $)^{10}$ to exercise training, with a systematic review of 11 randomised controlled trials (participants: $\mathrm{n}=1088$ ) reporting that up to $70 \%$ of individuals with CLBP do not complete prescribed home-based rehabilitation components. ${ }^{11}$ Factors associated with poor adherence to CLBP treatment include those reasons deemed person-related (resources, knowledge, attitudes, beliefs, perceptions and expectations of the person ${ }^{10}$; 
eg, motivation and distress), condition-related (particular illness-related demands faced by the person ${ }^{10}$; eg, pain intensity and disability) and therapy-related (treatment complexity, duration, prior failures, frequencies of change, immediacy of benefits, side effects and support ${ }^{10}$; $\mathrm{eg}$, supervision and intervention modality). ${ }^{11}$

Protection motivation theory (PMT) is an extension of the health belief model. ${ }^{12}$ PMT postulates that adherence to health behaviours is influenced by patient perceptions of the severity of the threat to their health, how effective the treatment is in addressing the threat and their self-efficacy towards completing the treatment. ${ }^{12}$ It has been demonstrated that the aforementioned factors of PMT are positively associated with one or more indices of home-based sport injury rehabilitation. ${ }^{13}$ Moreover, the belief in the effectiveness of, and adherence to, the programme has also been shown to impact rehabilitation. ${ }^{14} 15$ The application of PMT in the musculoskeletal pain rehabilitation setting is logical, given the rationale for seeking and sustaining treatment is borne from injury (ie, fear-arousing stimuli).${ }^{16}$ However, the factors of PMT have not been examined in individuals with CLBP. Therefore, the aim of our study was to explore the use of factors of PMT as a screening tool for adherence in participants with CLBP commencing a 6-month randomised clinical trial of general strength and condition compared with motor control and manual therapy.

\section{MATERIALS AND METHODS}

This study was a preplanned substudy of a larger 6-month randomised clinical trial that implemented two common non-surgical intervention approaches for the treatment of individuals with CLBP, from December 2015 to December 2016. ${ }^{17} 18$ Sample size calculations were based on the primary outcomes (lumbar intervertebral disc T2 time) of the larger randomised clinical trial. ${ }^{19}$ The full study protocol has been published and is summarised briefly as follows. ${ }^{19}$

\section{Patient and public involvement}

No patient or public involvement was sought.

\section{Participants}

Forty men and women aged 25-45 years with non-specific CLBP (>3 months) were recruited (figure 1). Exclusion criteria included (1) history of spinal surgery, (2) history of traumatic injury to the spine (eg, fracture and car accident), (3) symptoms of cauda equine syndrome, (4) planning surgery or invasive treatment (surgical injections) in the next 6 months, (5) known scoliosis, (6) symptoms of nerve root compression, (7) unable to communicate in English, (8) current treatment for CLBP, (9) reportedly engaging in more than $150 \mathrm{~min} /$ week moderatevigorous exercise, (10) participation in formal organised sport, (11) participation in gym-based exercise more than once per week, (12) having a compensable claim for CLBP, (13) pregnancy (or considering pregnancy in next 6 months or had given birth in last 9 months), (14) current smoker, (15) known anaemia, (16) body mass greater than $120 \mathrm{~kg}$, (17) family history of neurological or mental illness, and (18) implants unsuitable for MRI or dual-energy X-ray absorptiometry.

Participants were randomised to receive motor control exercise and manual therapy (MCMT, $n=20$ ), or general strength and conditioning (GSC, $\mathrm{n}=20$ ) by an offsite researcher with no contact with participants using block randomisation with random block lengths and stratification for gender prepared in advance using a web-based randomisation programme. MCMT involved 12 total 30 min one-on-one physiotherapy sessions over a 6 -month period. In the first 3-month period, 10 sessions (0-2 per week) were delivered, with 2 sessions in the final 3 months. Treatment was completed by qualified physiotherapists. Motor control exercises targeted transversus abdominis, multifidus and pelvic floor musculature. Progression was on a pain-contingent basis. ${ }^{20}$ Manual therapy was provided at the discretion of the physiotherapist and included posterior-anterior and transverse pressurebased intervention within the lumbar and pelvic regions. GSC involved 52 total 1 hour one-on-one supervised gymbased sessions. During the trial, participants attended two sessions per week (ie, 52 total). Sessions included aerobic and resistance exercises, progressed in a time-contingent manner Participants allocated to GSC were also asked to complete 20-40 min home-based aerobic exercise training three times per week over the 6-month study, as well as a $5-10 \mathrm{~min}$ mental rehearsal of movements associated with kinesiophobia during the first 6 weeks.

\section{Measures}

All measures were collected at baseline (before commencement of the interventional period) and 6 months (end of the interventional period).

The Sports Injury Rehabilitation Beliefs Scale (SIRBS) was used to assess the four components of PMT. ${ }^{13}$ The SIRBS is a 19-item scale with four factors: injury severity (5 items), susceptibility (5 items), treatment efficacy (4 items) and self-efficacy (4 items), plus a single item that measured the rehabilitation value (item: 'being fully recovered from injury is extremely important to me'). Items were scored on a 7-point Likert scale with endpoints of 'very strongly disagree' and 'very strongly agree'. Previously reported alpha coefficients were $0.52,0.84,0.85$ and 0.91 for injury severity, susceptibility, treatment efficacy and self-efficacy, respectively. ${ }^{13}$

The Positive and Negative Affect Schedule (PANAS) was used to assess affect. ${ }^{21}$ The scale consists of several words that describe different feelings and emotions. Participants were instructed to indicate to what extent they felt over the past week. Items were scored on a 5-point Likert scale with endpoints 'very slightly or not at all' and 'extremely'. Two 10-item mood scales comprise the PANAS with a possible range of 10-50 for each. A higher positive affect score indicates higher levels of positive affect and a lower negative affect score representing lower levels of negative 


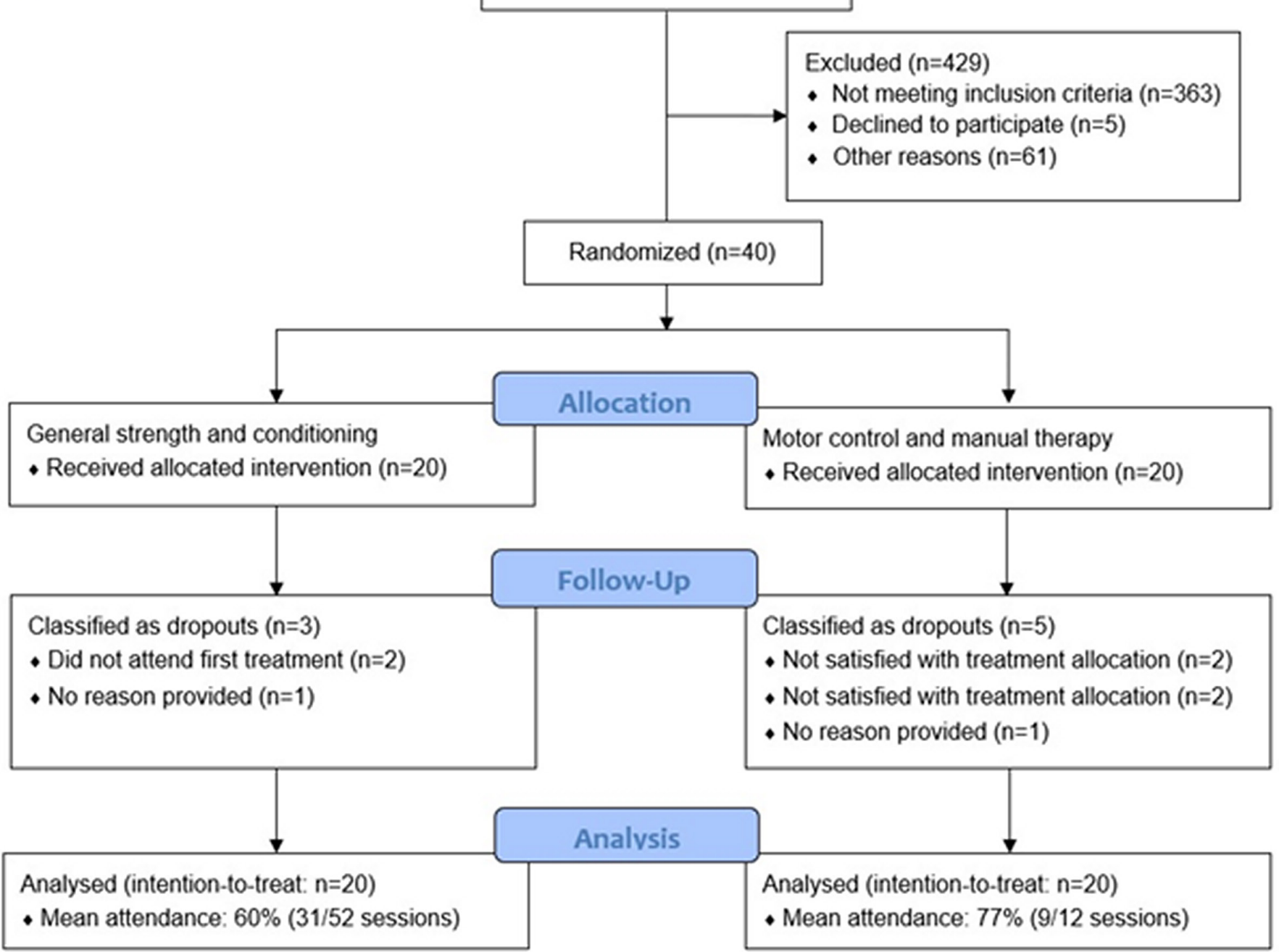

Figure 1 Consolidated Standards of Reporting Trials diagram.

affect. The scales are shown to be highly internally consistent, largely uncorrelated and stable at appropriate levels over a 2-month time period. ${ }^{21}$ The seven-item barriers checklist was implemented. ${ }^{16}$ Barriers included (1) lack of cooperation or support (from employer or family), (2) fear of reinjury, (3) not being able to pay for treatment or insurance restrictions, (4) fear of pain or further discomfort, (5) not having enough time, (6) having to care for child or family members and (7) scheduling problems. Participants responded either 'yes' or 'no' regarding the occurrence of the barriers, and a total score out of 7 was calculated.

\section{Adherence behaviour}

Attendance ratio (attendance and participation in sessions divided by total sessions) was calculated in line with established recommendations. ${ }^{22}$ Number of 'no shows' was defined as sessions in which the participant did not provide prior notification of cancellation, whereas sessions missed with notification were defined as cancellations. Reasons for cancellations included participant illness, injury or other commitments. If a participant was unable to attend a session yet completed a preplanned home-based session instead, we did not included this as a missed sessions. Dropout (yes/no) was defined as either formally withdrawing from the study or did not attend postintervention follow-up at 6 months.

Behaviour during each session was measured with the Sports Injury Rehabilitation Adherence Scale (SIRAS) for the GSC group only, which assessed therapist perceptions of face-to-face participant adherence. ${ }^{23}$ SIRAS is a three-item scale scored on a 5-point Likert scale. The three items were (1) intensity during competition of exercises ( $1=$ 'minimum effort' and 5='maximum effort'), (2) frequency of following instructions and advice ( $1=$ 'never' and $5=$ 'always') and (3) receptiveness to changes in the programme (1='very unreceptive' and 'very 5=receptive'). The previously reported alpha coefficient for the tool was $0.82{ }^{23}$ 


\section{Data analysis}

All analyses were conducted using Stata statistical software V.15. The strength and direction of associations between all variables were assessed by Spearman rank-order correlation coefficient. Separate stepwise multiple linear regression models were used to predict dependent variables (adherence ratio, number of no shows and number of cancellations) using independent variables (injury severity, susceptibility, treatment efficacy, self-efficacy, satisfactory rehabilitation, positive affect, negative affect and total barriers). Stepwise logistic regression models were used to predict dropout (yes/no) based on independent variables in both groups separately. An adjusted alpha level of 0.10 to enter and 0.20 to remove was used for the creation of all regression models based on previous research. ${ }^{24}$ SIRAS data were not considered for inclusion as a dependent variable in regression models due to the homogeneity of values (ie, no values other than five were observed). An alpha level of 0.05 was adopted for all other statistical tests unless stated otherwise.

\section{RESULTS \\ Demographics}

Forty participants $(\mathrm{GSC}=20, \mathrm{MCMT}=20)$ were included, with nine $(45 \%)$ and $10(50 \%)$ women in the GSC and MCMT groups, respectively. Mean ages were 35 (SD 5) and 35 (SD 4) years in the GSC and MCMT groups, respectively. Mean pain intensity was 43/100 (SD 20/100) points in the GSC group and 50/100 (17/100) points in the MCMT group. Mean attendance ratio was $77 \%$ (approx. $9 / 12$ sessions) and 60\% (approx. 31/52 sessions) in the MCMT and GSC groups, respectively. Perfect attendance was observed for $11(55 \%)$ participants in the MCMT group (ie, 12/12 sessions) and no participants in the GSC group (ie, 52/52 sessions). During each GSC session, the therapists observed no SIRAS values other than five across all three components (indicating maximum effort, following of instructions and receptiveness to changes in the programme). The mean numbers of cancellations and no shows in the GSC group were 6 (SD 4) and 15 (SD 16) sessions, respectively, and 1 (SD 3) and 1 (SD 3) sessions in the MCMT group, respectively. Eight $(\mathrm{GSC}=3$, MCMT $=5$ ) participants were classified as dropouts.

\section{SIRBS, PANAS and barriers}

Among the overall sample, mean values were 27.9 (SD 5.7) points for susceptibility, 18.6 (SD 4.7) points for treatment efficacy, 23.2 (SD 4.1) points for self-efficacy, 20.9 (SD 5.0) points for injury severity and 6.6 (1.2) points for rehabilitation value. Mean positive and negative affects were 31.9 (SD 5.5) and 19.5 (SD 5.4) points, respectively. The mean number of barriers was 2.9 (SD 1.8).

\section{Correlations}

Correlations between each variable examined in the overall cohort $(\mathrm{n}=40)$ are shown in table 1 . Treatment efficacy $(\mathrm{p}=0.019)$, self-efficacy $(\mathrm{p}=0.001)$, rehabilitation value $(\mathrm{p}=0.028)$ and injury severity $(\mathrm{p}=0.002)$ were positively correlated with susceptibility. Rehabilitation value also positively correlated with self-efficacy $(\mathrm{p}=0.005)$. Moreover, injury severity positively correlated with rehabilitation value $(\mathrm{p}=0.011)$. Finally, the number of no shows $(p<0.001)$, the number of cancellations $(p=0.005)$ and the dropout status $(\mathrm{p}<0.001)$ negatively correlated with the attendance ratio.

\section{Predicting attendance behaviour}

No independent variables met the criteria for inclusion in the final stepwise regression model for attendance ratio or number of no shows in the overall sample. However, the final model for number of cancellations included rehabilitation value only $\left(\mathrm{R}^{2}=0.117\right.$, adjusted $\mathrm{R}^{2}=0.093$, $\mathrm{F}_{1,37}=4.91, \mathrm{p}=0.033$ ); hence, approximately $12 \%$ of the

Table 1 Correlations between variables examined in the overall cohort $(\mathrm{N}=40)$

\begin{tabular}{|c|c|c|c|c|c|c|c|c|c|c|c|c|}
\hline \multirow[b]{2}{*}{ Variables } & \multicolumn{12}{|c|}{ Correlation for variable } \\
\hline & 1 & 2 & 3 & 4 & 5 & 6 & 7 & 8 & 9 & 10 & 11 & 12 \\
\hline 1. Barriers & - & & & & & & & & & & & \\
\hline 2. Susceptibility & -0.053 & - & & & & & & & & & & \\
\hline 3. Treatment efficacy & 0.218 & $0.375^{\star}$ & - & & & & & & & & & \\
\hline 4. Self-efficacy & -0.198 & $0.493^{\star \star}$ & 0.166 & - & & & & & & & & \\
\hline 5. Rehabilitation value & -0.115 & $0.352^{\star *}$ & 0.086 & $0.440^{\star *}$ & - & & & & & & & \\
\hline 6. Injury severity & 0.144 & $0.478^{\star *}$ & 0.265 & 0.292 & $0.404^{*}$ & - & & & & & & \\
\hline 7. Positive affect & 0.130 & 0.048 & 0.188 & -0.070 & -0.174 & 0.282 & - & & & & & \\
\hline 8. Negative affect & 0.245 & -0.061 & 0.234 & -0.164 & 0.008 & 0.007 & -0.134 & - & & & & \\
\hline 9. Attendance ratio & -0.213 & -0.097 & -0.196 & 0.065 & 0.179 & 0.070 & -0.187 & -0.272 & - & & & \\
\hline 10. Number of no shows & 0.150 & 0.114 & 0.025 & -0.195 & -0.254 & -0.052 & 0.205 & 0.180 & $-0.715^{\star \star \star}$ & - & & \\
\hline 11. Number of cancellations & 0.157 & 0.106 & 0.105 & -0.209 & -0.282 & -0.056 & 0.133 & 0.264 & $-0.437^{\star \star}$ & 0.262 & - & \\
\hline 12. Dropout status & -0.034 & -0.133 & -0.068 & -0.086 & -0.064 & -0.226 & -0.113 & 0.034 & $-0.615^{\star \star \star}$ & 0.246 & -0.073 & - \\
\hline
\end{tabular}

Data are Spearman's rho.

${ }^{*} \mathrm{P}<0.05,{ }^{* \star} \mathrm{P}<0.01,{ }^{* * *} \mathrm{P}<0.001$. 
variance in number of cancellations was accounted for by rehabilitation value.

In the GSC group, the final model for number of cancellations also included rehabilitation value only $\left(\mathrm{R}^{2}=0.200\right.$, adjusted $\left.\mathrm{R}^{2}=0.155, \mathrm{~F}_{1,18}=4.50, \mathrm{p}=0.048\right)$; therefore, rehabilitation value explained approximately $20 \%$ of the variance in number of cancellations. No independent variables met the criteria for inclusion in the final models for attendance ratio or number of no shows.

In the MCMT group, the final model for number of no shows included rehabilitation value and susceptibility $\left(\mathrm{R}^{2}=0.511\right.$, adjusted $\left.\mathrm{R}^{2}=0.450, \mathrm{~F}_{2,16}=8.35, \mathrm{p}=0.003\right)$; hence, greater than half the variance in number of no shows was accounted for by rehabilitation value and susceptibility. No independent variables met the criteria for inclusion in the final models for attendance ratio or number of cancellations.

\section{Predicting dropout status}

No independent variables met the criteria for inclusion in the final model for predicting dropout status in the overall cohort or either treatment group.

\section{DISCUSSION}

The main findings from this study were that perceptions of rehabilitation value (ie, the extreme importance of being fully recovered from injury) appeared to predict attendance behaviour in patients with CLBP. These observations also corresponded with the number of cancellations in the overall sample and GSC group, as well as the number of no shows among the MCMT group. Moreover, significant correlations were observed between perceptions of treatment efficacy, self-efficacy, rehabilitation value, injury severity and susceptibility. However, no factors of PMT predicted dropout status, and the SIRAS tool appeared to be limited in terms of differentiation of adherence behaviour in our study.

Our study demonstrated that perceived rehabilitation value predicted adherence behaviour, in terms of the number of cancellations, in patients with CLBP. These findings differ from previous applications of PMT in 85 patients recovering from anterior cruciate ligament knee reconstruction, in which treatment efficacy and selfefficacy were shown to predict adherence. ${ }^{23}$ Our results align with data from 62 student athletes that demonstrated the importance of rehabilitation value on adherence. ${ }^{13}$ Of note, our model accounted for approximately only $12 \%$ of the variance in number of cancellations, and thus it is worth considering additional factors that may predict adherence. For example, treatment-based (eg, patient-centred approach or intensity of therapy) and environment-based (eg, architecture or interior design) contextual factors surrounding the clinical encounter warrant further investigation. ${ }^{25}$ Our findings highlight the importance of patients believing they can achieve full recovery from the condition, given this appears to predict treatment adherence. However, it is important that patients are aware that the management of CLBP is often ongoing rather than curative. While potentially disheartening to patients, erroneous expectations of achieving a full recovery have been shown to hinder the management of this condition. ${ }^{26}$ Therefore, ensuring patients commencing a rehabilitation programme have realistic expectations may be imperative to its success.

To our knowledge, our study is the first to consider the four factors of PMT, as assessed by SIRBS, in patients with CLBP. Notably, coping appraisal (treatment efficacy and self-efficacy) correlated with susceptibility, which suggests these beliefs may be relevant to target during the rehabilitation process for CLBP. Moreover, injury severity correlated with susceptibility, which underscores the importance of establishing CLBP as a manageable condition to avoid beliefs detrimental to its treatment. ${ }^{26}$ While application of PMT remains in its infancy in clinical population groups, such as those with CLBP, our observations highlight beliefs that should be considered when designing future interventions and implementing current treatment.

Collectively, eight (20\%) participants dropped out during the interventional period of our study, although measures of PMT were unable to predict these behaviours. In contrast, a previous study that examined 229 participants completing short-term rehabilitation across a range of physical injuries found affect, injury severity and self-efficacy predicted dropout status. ${ }^{16}$ Differences may in part be explained by patient demographics. The previous study enrolled patients undergoing 4-8 weeks of rehabilitation and noted this aimed at avoiding those with chronic pathologies and the associated complexities. ${ }^{16}$ Therefore, variables that predict dropout in acute and subacute conditions may not be applicable in chronic conditions, such as that required for CLBP. Another consideration is the context in which the adherence behaviours were being assessed. The participants from the current study are part of a randomised clinical trial and therefore perhaps feel beholden to continue irrespective of their perception of the severity of the threat to their health, how effective the health behaviour is in addressing the threat and how capable they are to perform the required health behaviour.

SIRAS has previously been shown to be a reliable measure of clinic-based adherence to physiotherapy rehabilitation in patients with musculoskeletal injuries. ${ }^{27}$ However, the application of SIRAS as an assessment of adherence behaviour during each GSC treatment session appeared limited in our cohort of patients with CLBP. As with any intervention-based study, particularly those involving exercise training, volunteer bias may have been apparent. This may have resulted in the enrolment of patients with CLBP with a penchant for exercise training, and thus these participants would be more likely to adhere, when compared with those in the wider rehabilitation setting, when randomised to this intervention. Our 
observations suggest that while SIRAS may be reliable in the clinical-based setting, it may have limitations in the research-based setting.

This study was strengthened by the pragmatic design of the interventions, whereby both treatments reflected interventions used in the management of CLBP. ${ }^{28}$ Therefore, findings associated with adherence behaviour in our study may be extrapolated to the rehabilitation setting. However, limitations of the current study should be considered. First, adherence to home-based components of these interventions was not considered, which may have influenced face-toface session adherence. Second, as the population examined had chronic pain, dropout status and/or adherence behaviours may have been influenced by fluctuations in pain that were not detected during routine assessment of pain intensity. Third, SIRAS data was only collected in the GSC group, hence conclusions drawn on its potential limitations do not also expand to MCMT. Finally, prediction models were considered retrospectively; therefore, we were unable to conclude whether addressing significant independent variables prospectively influences adherence behaviours.

This study demonstrated that the perceived value of rehabilitation may in part predict adherence behaviour in patients with CLBP. Moreover, relationships between varying factors of PMT were observed in this clinical population group. Future research would benefit from exploration of additional contextual factors associated with the clinical encounter. Given the findings of the current study, clinicians in the rehabilitation setting should consider these patient beliefs during treatment (eg, when establishing patient-centred goals) to address adherence-based concerns.

Twitter Patrick J Owen @PatrickOwenPhD, Luana C Main @LuanaCMain, Jon J Ford @stopsbackpain and Daniel L Belavy @belavyprof

Acknowledgements The authors thank the participants for taking part in the study and their colleagues on the wider study. The authors also thank Professor Jeremy Lewis for feedback on the manuscript prior to submission.

Contributors Study design: LCM, CTM, JJF, AJH and DLB. Data analysis: PJO. Data interpretation and drafting of the manuscript: PJO and LCM. Study conduct, data collection, revision of manuscript content and approval of the final version of the manuscript: all authors. PJ0 accepts full responsibility for the finished work and/ or the conduct of the study, had access to the data, and controlled the decision to publish.

Funding This work was supported by Deakin University (award/grant number: not applicable) under internal funding (to DB).

Competing interests None declared.

Patient and public involvement Patients and/or the public were not involved in the design, conduct, reporting or dissemination plans of this research.

Patient consent for publication Consent obtained directly from patient(s)

Ethics approval This study involves human participants, was approved by Deakin University Human Research Ethics Committee and registered with the Australian New Zealand Clinical Trials Registry (date registered 20 November 2015) and complied with the Declaration of Helsinki. Participants gave informed consent to participate in the study before taking part.

Provenance and peer review Not commissioned; externally peer reviewed.
Data availability statement Data are available upon reasonable request. Data sharing statement: The data that support the findings of this study are available from the corresponding author upon reasonable request.

Open access This is an open access article distributed in accordance with the Creative Commons Attribution Non Commercial (CC BY-NC 4.0) license, which permits others to distribute, remix, adapt, build upon this work non-commercially, and license their derivative works on different terms, provided the original work is properly cited, appropriate credit is given, any changes made indicated, and the use is non-commercial. See: http://creativecommons.org/licenses/by-nc/4.0/.

\section{ORCID iDs}

Patrick J Owen http://orcid.org/0000-0003-3924-9375

Luana C Main http://orcid.org/0000-0002-9576-9466

Clint T Miller http://orcid.org/0000-0001-7743-6986

Jon J Ford http://orcid.org/0000-0001-5524-190X

Andrew J Hahne http://orcid.org/0000-0002-6227-5339

Daniel L Belavy http://orcid.org/0000-0002-9307-832X

\section{REFERENCES}

1 Hoy D, March L, Brooks $\mathrm{P}$, et al. The global burden of low back pain: estimates from the global burden of disease 2010 study. Ann Rheum Dis 2014;73:968-74.

2 Vos T, Flaxman AD, Naghavi M, et al. Years lived with disability (YLDs) for 1160 sequelae of 289 diseases and injuries 1990-2010: a systematic analysis for the global burden of disease study 2010. Lancet 2012;380:2163-96.

3 Koes BW, van Tulder M, Lin C-WC, et al. An updated overview of clinical guidelines for the management of non-specific low back pain in primary care. Eur Spine J 2010;19:2075-94.

4 Hoy D, Brooks P, Blyth F, et al. The epidemiology of low back pain. Best Pract Res Clin Rheumatol 2010;24:769-81.

5 Hashemi L, Webster BS, Clancy EA, et al. Length of disability and cost of work-related musculoskeletal disorders of the upper extremity. J Occup Environ Med 1998;40:261-9.

6 Walker BF, Muller R, Grant WD. Low back pain in Australian adults: the economic burden. Asia Pac J Public Health 2003;15:79-87.

7 Maher C, Underwood M, Buchbinder R. Non-specific low back pain. Lancet 2017;389:736-47.

8 Owen PJ, Miller CT, Mundell NL, et al. Which specific modes of exercise training are most effective for treating low back pain? Network meta-analysis. Br J Sports Med 2020;54:1279-87.

9 Owen PJ, Miller CT, Mundell NL, et al. Infographic. What kinds of exercise are best for chronic low back pain? Br J Sports Med 2020;54:1231-2.

10 World Health Organization. Adherence to long-term therapies: evidence for action. Geneva: World Health Organization, 2003.

11 Beinart NA, Goodchild CE, Weinman JA, et al. Individual and intervention-related factors associated with adherence to home exercise in chronic low back pain: a systematic review. Spine $J$ 2013;13:1940-50.

12 Rogers RW. A protection motivation theory of fear appeals and attitude change. J Psychol 1975;91:93-114.

13 Taylor AH, May S. Threat and coping appraisal as determinants of compliance with sports injury rehabilitation: an application of protection motivation theory. J Sports Sci 1996;14:471-82.

14 Duda JL, Smart AE, Tappe MK. Predictors of adherence in the rehabilitation of athletic injuries: an application of personal investment theory. J Sport Exerc Psychol 1989;11:367-81.

15 Noyes FR, Matthews DS, Mooar PA, et al. The symptomatic anterior cruciate-deficient knee. Part II: the results of rehabilitation, activity modification, and counseling on functional disability. J Bone Joint Surg Am 1983;65:163-74.

16 Grindley EJ, Zizzi SJ, Nasypany AM. Use of protection motivation theory, affect, and barriers to understand and predict adherence to outpatient rehabilitation. Phys Ther 2008;88:1529-40.

17 Owen PJ, Miller CT, Rantalainen T, et al. Exercise for the intervertebral disc: a 6-month randomised controlled trial in chronic low back pain. Eur Spine J 2020;29:1887-99.

18 Tagliaferri SD, Miller CT, Ford JJ. Randomized trial of general strength and conditioning versus motor control and manual therapy for chronic low back pain on physical and self-report outcomes. $J$ Clin Med 1726;2020:9.

19 Simson KJ, Miller CT, Ford J, et al. Optimising conservative management of chronic low back pain: study protocol for a randomised controlled trial. Trials 2017;18:184. 
20 Macedo LG, Latimer J, Maher CG, et al. Effect of motor control exercises versus graded activity in patients with chronic nonspecific low back pain: a randomized controlled trial. Phys Ther 2012;92:363-77.

21 Watson D, Clark LA, Tellegen A. Development and validation of brief measures of positive and negative affect: the PANAS scales. $J$ Pers Soc Psychol 1988;54:1063-70.

22 American College of Sports Medicine. ACSM's guidelines for exercise testing and prescription. 11th edn. Philadelphia: Wolters Kluwer, 2021.

23 Brewer BW, Van Raalte JL, Petitpas AJ, et al. Preliminary psychometric evaluation of a measure of adherence to clinic-based sport injury rehabilitation. Physical Therapy in Sport 2000;1:68-74.
24 Belavy DL, Owen PJ, Livingston PM. Do successful PhD outcomes reflect the research environment rather than academic ability? PLoS One 2020;15:e0236327.

25 Miller CT, Owen PJ, Than CA. Attempting to separate placebo effects from exercise in chronic pain: a systematic review and meta-analysis. Sports Med 2021. doi:10.1007/s40279-021-01526-6

26 Main CJ, Foster N, Buchbinder R. How important are back pain beliefs and expectations for satisfactory recovery from back pain? Best Pract Res Clin Rheumatol 2010;24:205-17.

27 Kolt GS, Brewer BW, Pizzari T, et al. The sport injury rehabilitation adherence scale: a reliable scale for use in clinical physiotherapy. Physiotherapy 2007:93:17-22

28 Belavy DL, Diwan AD, Ford J, et al. Network meta-analysis for comparative effectiveness of treatments for chronic low back pain disorders: systematic review protocol. BMJ Open 2021;11:e057112. 